\title{
Formal support from senior nursing management results in improved nutrition screening tool completion rates
}

\author{
M. Gibbs ${ }^{1}$ and C. E. Weeks ${ }^{2}$ \\ ${ }^{1}$ School of Biomedical \& Health Sciences, King's College London, Stamford Street, London SE1 9NH, UK \\ and ${ }^{2}$ Department of Nutrition \& Dietetics, Guy's \& St Thomas' NHS Foundation Trust, Westminster Bridge Road, \\ London SE1 7EH, UK
}

\begin{abstract}
A validated nutrition screening tool (NST) was launched in an acute hospital Trust in 2000. In 2006 the Trust Nutrition Steering Committee made NST completion mandatory for all inpatients, to comply with NICE guidelines ${ }^{(1)}$. Audits of NST completion rates conducted in 2000, 2003 and 2007 showed a completion rate of approximately $35 \%$ in each audit (see Table), despite staff training and other strategies designed to improve screening. Since 2007 nurse managers have been required to report monthly to the Director of Nursing and their peers on screening and weekly re-screening rates on their wards. The aim of the present study was to determine whether this initiative resulted in improved NST completion rates.

The audit was conducted on four wards, three of which had been audited previously. Each ward was audited for 1 week. The end-of-bed charts of all patients were reviewed and the number of NST completed (partially or fully) on admission was recorded. $\chi^{2}$ tests were conducted to test for differences in NST completion rates between the audits.
\end{abstract}

\begin{tabular}{lccccc}
\hline & $\begin{array}{c}\text { Audit 1 } \\
\text { (May 2000) }\end{array}$ & $\begin{array}{c}\text { Audit 2 } \\
\text { (February 2003) }\end{array}$ & $\begin{array}{c}\text { Audit 3 } \\
\text { (June 2007) }\end{array}$ & $\begin{array}{c}\text { Audit 4 } \\
\text { (June 2008) }\end{array}$ & $P$ \\
\hline Wards $(n)$ & 11 & 9 & 8 & 4 & - \\
Patients $(n)$ & 485 & 216 & 128 & 187 & - \\
NST completed & & & & & \\
$n$ & 184 & 78 & 41 & 113 & $<0.01$ \\
$\%$ & 38 & 36 & 32 & 60 & \\
\hline
\end{tabular}

The percentage of NST completed on admission, at least partially, almost doubled in 2008 compared with previous audits. However, only sixty-five $(35 \%)$ were fully completed. In the three wards audited previously completion rates increased significantly on two (elderly care, $7-15 \%$ in previous audits to $84 \%$ in $2008(P<0.01)$; general medical, $0-35 \%$ in previous audits to $81 \%$ in $2008(P<0.01))$. The other ward (acute admissions) increased from approximately $60 \%$ in all previous audits to $75 \%$ in $2008(P=0.231)$. In the ward that had not been audited previously (cardiac) NST completion rate was $22 \%(n$ 13). In audit 4 it was noted that females $(n 58 ; 74 \%)$ were significantly more likely to be screened than males $(n 55 ; 50 \% ; P=0.001)$, which suggests an element of selection in who is screened and who is not.

The study suggests admission NST completion rates can be improved substantially with the formal support of senior nursing management. In this Trust this strategy was more successful in increasing completion rates than previous initiatives. However, there continues to be a failure to recognise the need to screen all patients and to complete the NST fully.

1. National Institute for Health and Clinical Excellence (2006) Nutrition Support in Adults: Oral Nutrition Support, Enteral Tube Feeding and Parenteral Nutrition. Clinical Guideline 32. London: NICE. 